\title{
Novel aspects of eicosanoid signaling through the use of gene-targeted mice
}

\author{
Ying Yu' and Colin D. Funk ${ }^{2}$
}

I Institute for Translational Medicine and Therapeutics, University of Pennsylvania, Philadelphia, Pennsylvania, USA;

${ }^{2}$ Departments of Biochemistry and Physiology, Queen's University, Kingston, Ontario, Canada

Abstract

Eicosanoids are lipid mediators consisting of prostaglandins, leukotrienes, lipoxins and related compounds derived primarily from arachidonic acid and to a lesser extent from eicosapentaenoic and di-homo-gammalinolenic acids. This large class of bioactive lipids is derived from the initial release of polyunsaturated fatty acid from the sn2 position of glycerophospholipids predominantly from cytosolic phospholipase $\mathrm{A}_{2}$ and subsequent conversion by either prostaglandin H synthases-1 and -2 (PGHS-1, PGHS-2; also known as COX1, COX-2), lipoxygenases or various members of the cytochrome P450 family. Eicosanoids control a vast array of physiological functions, including female reproductive function and parturition, platelet aggregation and vascular homeostasis, as well as renal function and roles in inflammation initiation and resolution. The authors have been studying the functions and signaling of eicosanoids, in particular the prostaglandin class of molecules, using a series of induced mutant mouse strains created in the laboratory by manipulation of the PGHS-1 and PGHS-2 genes by gene targeting in embryonic stem cells. The two strains of mice most fully characterized to date are referred to as "low-dose aspirin genetic mimic" or PGHS-1 knockdown and "selective COX-2 inhibitor genetic mimic" or PGHS-2 Y385F. This brief review describes the utility of these mouse models for unraveling new insights into eicosanoid signaling.

Keywords: aspirin; cardiovascular; coxib; inflammation; prostaglandin

Abbreviations: AA: arachidonic acid; ASA: aspirin; BP: blood pressure; COX: cyclooxygenase; DA: ductus arteriosus; IEL: internal elastic lamina; KD: knockdown; KO: knockout; LOX: lipoxygenase; NSAID: nonsteroidal anti-inflammatory drug; PG: prostaglandin; $P G H S$ : prostaglandin $H$ synthase; $P_{G I}$ : prostacyclin; POX: peroxidase; TPA: tetradecanoyl phorbol acetate; Tx $A_{2}$ : thromboxane $A_{2}$; WT: wild-type.

\section{Introduction}

$\mathrm{T}$ here are three major enzymic pathways of eicosanoid biosynthesis via dietary essential fatty acid-catalyzed conversion by prostaglandin $\mathrm{H}$ synthases (PGHS), lipoxygenases (LOX) and cytochromes P450. The PGHS pathways produce prostaglandins (PGs), prostacyclin $\left(\mathrm{PGI}_{2}\right)$ and thromboxane $\mathrm{A}_{2}\left(\mathrm{TxA}_{2}\right)$, lipoxygenase pathways give rise to leukotrienes, lipoxins, and 5-, 12and 15-hydroxy-eicosatetraenoic acids, while the cytochrome P450 pathway metabolites are hydroxylated or epoxy-metabolites of arachidonic acid (AA). These eicosanoids possess a vast array of biological functions in pain, inflammation and many disease settings. The focus of this short review is to illuminate new understanding of the oldest class of eicosanoids, e.g. prostaglandins, by using gene-manipulated mice to alter the expression of PGHS.
Non-steroidal anti-inflammatory drugs (NSAIDs) target PGHS enzymes, which exist in two isoforms, PGHS-1 and PGHS-2. Aspirin and other traditional NSAIDs in the class are nonselective inhibitors of both PGHS-1 and PGHS-2, while newer agents termed "coxibs", including rofecoxib, valdecoxib and celecoxib, are selective inhibitors of PGHS-2. Both PGHS isozymes are bifunctional with a cyclooxygenase (COX) activity and a functional linked peroxidase (POX) activity. The NSAIDs, including aspirin and coxibs, act via inhibition of the COX activity, while leaving POX activity intact. Aspirin is the most widely used drug in the world, and low-dose aspirin use is effective in the secondary prevention of both heart attack and stroke (1-3) and in the primary prevention of coronary heart disease in at-risk individuals (4-6). The development of coxibs was based on the notion that PGHS-2 was the major source of $\mathrm{PGE}_{2}$ and 
$\mathrm{PGI}_{2}$ (prostacyclin), which mediates inflammation, and that PGHS-1 was the dominant PG source for gastric cytoprotection (7). The authors recently created two novel mouse strains, referred to as "low-dose aspirin genetic mimic" or PGHS-1 knockdown (KD) (8) and "selective COX-2 inhibitor genetic mimic" or PGHS-2 Y385F $(9,10)$ for the study of prostaglandin functions.

\section{Prostaglandin H synthase-I knockdown mouse model mimics low-dose aspirin treatment}

Insertion of a neomycin resistance cassette within intronic sequences has been reported previously to generate a hypomorphic allele or "knockdown" of gene expression to very low levels $(11-13)$. The PGHS-1 KD mice were generated by insertion of this cassette into intron 10 of the PGHS-1 gene through homologous recombination in murine embryonic stem cells. Follow-up characterization of the resultant mice proved that $\mathrm{PGE}_{2}$ production was diminished by $75 \%$ compared with wild-type (WT) in unstimulated peritoneal macrophages and PGHS-1 expression was suppressed similarly $(70-73 \%)$ in the macrophages and other PGHS-1 KD mouse tissues tested as well, which indicates that this model is globally hypomorphic for PGHS1 expression.

The PGHS-1 isoform, but not PGHS-2, is expressed in mature human platelets, which are the dominant source of $\mathrm{TxA}_{2}$ biosynthesis in humans (14). Pharmacological studies suggest that inhibition of the capacity of human platelets to generate $\mathrm{TxA}_{2}$ by more than $95 \%$ is necessary to accomplish inhibition of $\mathrm{TxA}_{2}$-dependent platelet aggregation ex vivo (15). The plasma thromboxane metabolite, $\mathrm{TxB}_{2}$, was depressed by $97 \%$ in PGHS- 1 KD mice compared with WT controls, which is remarkably similar to the inhibition achieved by low-dose aspirin treatment in mice $(96 \%)$ and agrees with the level of inhibition achieved in humans (15). Moreover, the platelets from both PGHS-1 KD and low-dose aspirin-treated WT mice (ASA/WT) failed to aggregate in response to various concentrations of AA that were effective in WT platelets (Fig. 1A). The PGHS-1 KD mouse model mimics low-dose aspirin clinical treatment regarding platelet function.
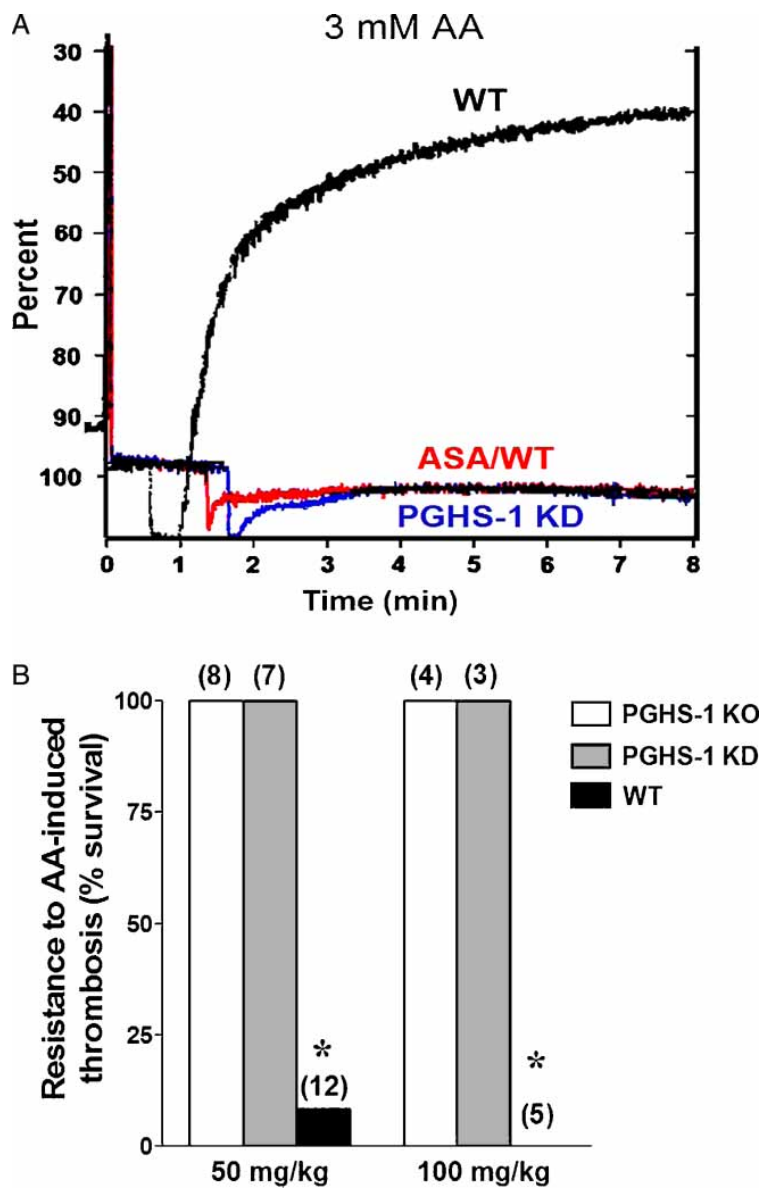

Fig. 1. Prostaglandin H synthase-1 (PGHS-1) knockdown (KD) mice represent a genetic mimic of low-dose aspirin (ASA) treatment in platelet aggregation and thrombosis studies. (A) Representative platelet aggregation tracings for PGHS-1 KD, wild-type (WT) and low-dose aspirin-treated WT mice (ASA/WT) at $3 \mathrm{mM}$ arachidonic acid (AA) concentration. (B) Both PGHS-1 KD and knockout (KO) mice are more resistant to thrombosis elicited by intravenous AA injection. Percentage survival of each group is indicated $\left(^{*} p<0.001\right.$, as determined by Fisher's exact test) and number of mice studied is in parentheses. Data are from Yu et al. (8).

\section{Protection against thrombosis and impaired inflammatory response in prostaglandin $\mathbf{H}$ synthase-I knockdown mice}

Since platelets from PGHS-1 KD mice have defective generation of thromboxane and impaired aggregation responses ex vivo, the finding that PGHS-1 KD mice were resistant to AA-induced thrombosis in vivo (Fig. 1B), while nearly all the normal WT controls died at the tested AA doses (8), could perhaps have been anticipated. However, since the time to arterial occlusion by clot formation was markedly prolonged in PGHS-1 KD mice compared with WT mice in a model of oxidative stress-induced arterial photochemical injury (8), the 
implications for thromboxane involvement in thrombosis are far reaching.

The role of PGHS-1-derived prostaglandins and low-dose aspirin in the setting of inflammation remains controversial. Several inflammatory models, including chemically evoked ear swelling assays by AA, capsaicin, phorbol esters and croton oil, and carrageenan-induced paw edema, were tested in PGHS-1 KD mice (8). PGHS-1 KD mice displayed a notably reduced inflammatory response to AA, capsaicin and carrageenan, but failed to demonstrate significant inflammatory protection in response to tetradecanoyl phorbol acetate (TPA) and croton oil, suggesting that PGHS-1-derived eicosanoids may be involved in some aspects of the inflammatory response in mice in addition to the well-known actions of PGHS-2-derived eicosanoids.

\section{Normal parturition in prostaglandin $\mathbf{H}$ synthase-I knockdown and low-dose aspirin- treated mice, but not in prostaglandin $\mathrm{H}$ synthase-I null mice}

During late gestation, PGHS-1 expression is upregulated in the uterus then decreases to undetectable levels after delivery (16), fitting with its requirement for normal initiation of parturition in mice. PGHS-1-derived $\mathrm{PGF}_{2 \alpha}$ mediates the initiation of parturition, and exogenous administration of this eicosanoid can rescue the parturition defect observed in PGHS-1 null mice (16) and induce a decline in serum progesterone to initiate the birthing process (17). Prostaglandin F receptor-deficient mice exhibit parturition failure owing to impaired withdrawal of serum progesterone at term (18). Uterus PGHS- 1 expression in PGHS-1 KD mice was about $12 \%$ of normal at day 17.5 of gestation, and only $8 \% \mathrm{PGF}_{2 \alpha}$ in these mice sufficed to prepare for normal labor onset at day 19 of gestation (Table 1). By contrast, the residual synthetic capacity in PGHS-1 null mice $\left(1.1 \% \mathrm{PGF}_{2 \alpha}\right)$ was unable to initiate normal parturition at term. Moreover, just before delivery at day 19 , the striking serum progesterone fall accompanied by oxytocin induction in myometrium was observed in PGHS-1 KD mice as in WT mice. Similarly to PGHS-1 KD mice, low-dose aspirin treatment to WT mice (ASA/ WT) did not affect initiation of full-term labor (Table 1). Use of these novel PGHS-1 KD mice has allowed an efficient titration of the amounts of prostaglandin necessary to achieve an important regulatory physiological process.

\section{Prostaglandin H synthase-2 Y385F mice represent a novel genetic mimic of coxib administration}

Tyrosine 385 of PGHS is a critical residue for initiation of COX catalysis, and the phenylalanine385 (Y385F) mutant of PGHS lacks COX activity but retains POX activity $(7,19)$. [Note that in this review, the tyrosine residue of PGHS-2 is numbered in parallel with ovine PGHS-1, the recognized standard in numbering nomenclature of PGHS isoforms to facilitate comparison of important residues in catalytic function. Accordingly, the actual position in mouse PGHS-2 is Tyr-371 based on numbering the amino-terminal methionine of the signal peptide as residue number 1.] To generate a mouse model that might mimic coxib administration, a Y385F point mutation was inserted into the PGHS-2 gene (known as Ptgs2) through a genetargeting strategy (10). Biochemical studies in vitro confirmed that peritoneal macrophages of PGHS-2 Y385F mice lack COX activity from inducible PGHS-2 protein without disruption of POX activity, which apparently replicates the scenario with selective PGHS-2 inhibitor (coxib) treatment (7).

Table I. Normal parturition in PGHS-I knockdown (KD) and low-dose aspirin-treated mice, but not in PGHS-I knockout (KO) mice

\begin{tabular}{|c|c|c|c|c|c|}
\hline Maternal genotype & Paternal genotype & No. & Labor onset (days) [range] & Average litter & Survival for $24 \mathrm{~h}(\%)$ \\
\hline PGHS-I KD & PGHS-I KD & 8 & $19.6 \pm 0.2[19.0-20.5]$ & $7.3 \pm 1.1$ & 90.0 \\
\hline $\mathrm{ASA} / \mathrm{WT}^{\mathrm{a}}$ & WT & 6 & $19.3 \pm 0.1[18.0-19.5]$ & $8.4 \pm 1.6$ & 92.3 \\
\hline PGHS-I KO & PGHS-I KO & 10 & $21.1 \pm 0.3^{* * *}[20.0-22.5]$ & $8.7 \pm 0.7$ & $54.0 *$ \\
\hline WT & WT & 7 & $19.5 \pm 0.2[19.0-20.0]$ & $6.8 \pm 0.5$ & 89.5 \\
\hline
\end{tabular}

Data from Yu et al. (8).

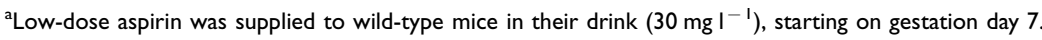

PGHS: prostaglandin $\mathrm{H}$ synthase; ASA: aspirin; WT: wild-type.

$*_{* *} *_{p}<0.001, *_{p}<0.05$ vs PGHS-I KD, ASA/WT and WT mating. 
$\mathrm{TxA}_{2}$ is the major PGHS-1 product from platelets, while $\mathrm{PGI}_{2}$ is synthesized dominantly from PGHS-2, which is expressed in sheer-stimulated endothelium (20). Coxibs can selectively suppress circulating prostacyclin in humans (21). Urinary thromboxane metabolite excretion is not affected by Y385F mutation in PGHS-2 Y385F mice. In contrast, prostacyclin metabolite excretion is dramatically diminished, which is similar to celecoxibtreated mice (9). PGHS-2 Y385F mouse appear to represent a genuine genetic model of coxib administration in mice.

\section{Elevated blood pressure and thrombosis susceptibility in prostaglandin $\mathbf{H}$ synthase-2 Y385F mice}

NSAIDs, including PGHS-2 selective inhibitors, have been associated with hypertension reports or deterioration of blood pressure (BP) control in patients receiving antihypertensive drugs $(22,23)$, and this hypertension shows a relationship to both selectivity of PGHS-2 inhibition and duration of drug exposure (24). Short-term studies of PGHS-2 inhibitors reveal that they evoke transient sodium retention (25). As expected, elevated $\mathrm{BP}$, including both diastolic and systolic measures, was observed in PGHS-2 Y385F mutant and PGHS-2 selective inhibitor celecoxib-treated mice on a regular chow diet compared with WT controls (Fig. 2A).

Prostacyclin is the predominant PG product in endothelium, causing vasodilatation and inhibition of platelet aggregation, and preventing the proliferation of vascular smooth-muscle cells in vitro. In contrast, thromboxane has opposing actions, evoking platelet aggregation, vasoconstriction and vascular proliferation (20). Since the balance of these two eicosanoids was disrupted as measured by urinary metabolites, as mentioned above, the cardiovascular effects of thromboxane would be expected to be exaggerated. Consistent with these observations, genetically induced PGHS-2 inhibition in PGHS-2 Y385F mice augments the thrombotic response to the thromboxane agonist U46619 (Fig. 2B), and a highly selective PGHS-2 inhibitor, DFU, increases thrombosis susceptibility in a photochemical vascular injury model (9).
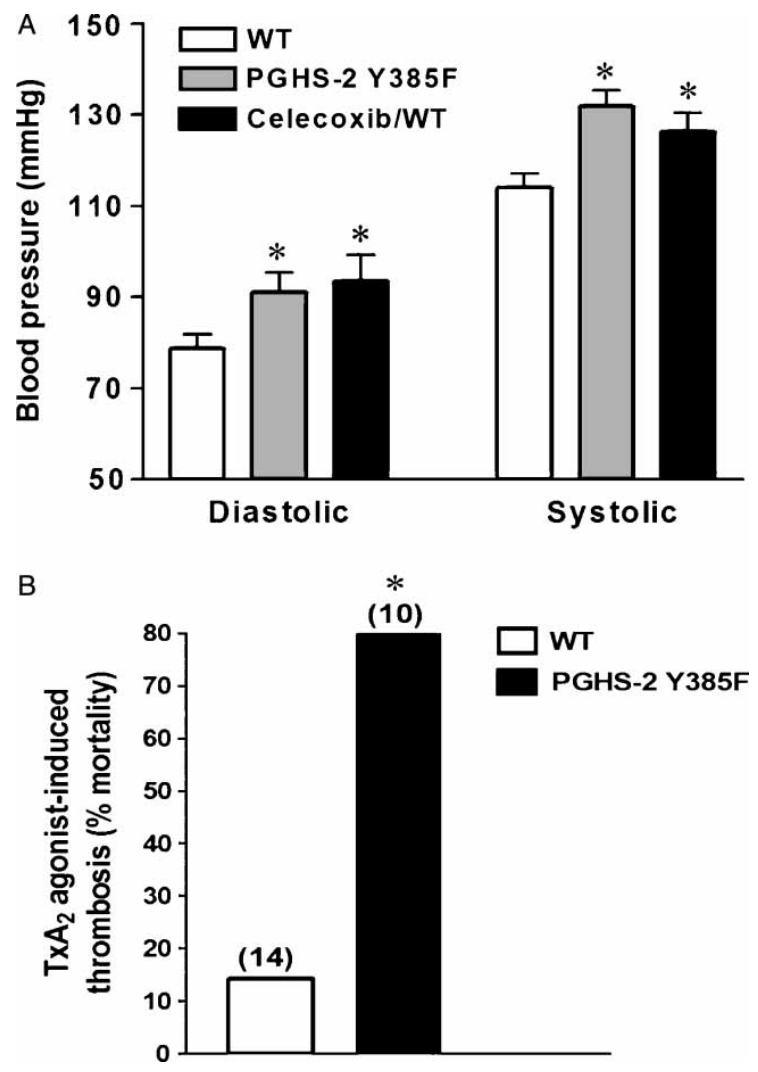

Fig. 2. Both genetic disruption and pharmacological inhibition of COX activity of prostaglandin $\mathrm{H}$ synthase-2 (PGHS-2) increase blood pressure and susceptibility to thrombosis. (A) Blood pressure (BP) measurement by tail cuff method. Both diastolic and systolic BP were elevated significantly in PGHS-2 Y385F and celecoxibtreated mice compared with wild-type (WT) mice $\left({ }^{*} p<0.01\right.$, $n=10-12$ ). (B) Thrombotic challenge in WT and PGHS-2 Y385F mice. Data represent sudden death percentage after injection of $0.2 \mathrm{mg} \mathrm{kg}^{-1}$ thromboxane analog U46619; the mortality of PGHS-2 $\mathrm{Y} 385 \mathrm{~F}$ mice is significantly increased compared with WT mice $\left({ }^{*} p<0.01, n=10-14\right)$. TxA $\mathrm{A}_{2}$ : thromboxane $\mathrm{A}_{2}$.

Normal neonatal ductus arteriosus remodeling in prostaglandin $\mathrm{H}$ synthase- $2 \mathrm{Y} 385 \mathrm{~F}$, but not in prostaglandin $\mathbf{H}$ synthase- 2 null mice

The fetal ductus arteriosus (DA) is an essential conduit for deoxygenated blood to bypass the collapsed lungs to enter via the descending aorta to the placenta. PGs are essential mediators of DA patency during fetal life and can modulate its constriction in the neonatal period (26). Mice deficient in PGHS-2 $(10,27)$ or lacking the PG receptor EP4 (28) predispose to failure of DA remodeling at birth. Surprisingly, patent DA and resulting neonatal mortality were not observed in PGHS-2 Y385F mice and celecoxib-treated mice (Fig. 3). Electron microscopic analysis of the DA $4 \mathrm{~h}$ after delivery revealed multiple endothelial cell layers oriented toward the lumen, fragmentation of 

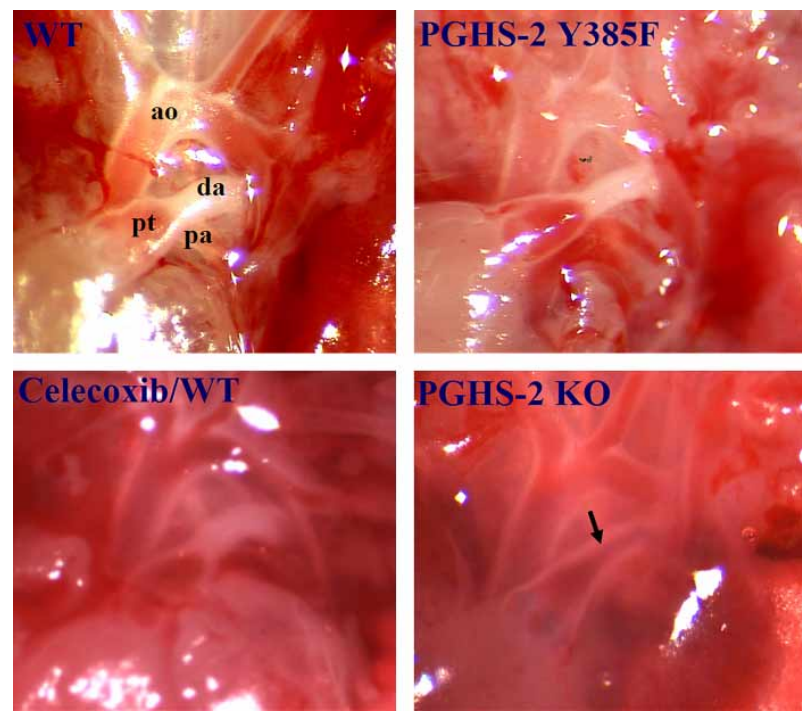

Fig. 3. Representative morphological examination $(15 \times$ magnification) of ductus arteriosus in neonatal wild-type (WT), prostaglandin $\mathrm{H}$ synthase-2 (PGHS-2) Y385F, PGHS-2 knockout (KO) and celecoxib-treated mice. Celecoxib/WT: WT pregnant females fed a diet containing $800 \mathrm{ppm}$ celecoxib at gestation day 7. ao: aorta; pt: pulmonary track; pa: pulmonary artery; da: ductus arteriosus; arrow: patent ductus arteriosus. Data are from $\mathrm{Yu}$ et al. (10).

the internal elastic lamina (IEL) and smooth-muscle cell infiltration to the subendothelial region across the fragmented IEL in PGHS-2 Y385F and WT mice, indicating successful remodeling. In contrast, the patent DA in PGHS-2 null mice display a single layer of endothelial cells with an intact IEL. This normal DA remodeling in PGHS-2 Y385F mutant mice at birth might be explained by a novel means of PGHS-1/PGHS-2 heterodimer signaling in the DA at birth (10). Thus, molecular modeling and a variety of biochemical tests provided evidence that PGs can be synthesized from an active PGHS-1/ mutant PGHS2 complex and that the two PGHS isoforms can associate (10).

\section{Summary}

Both PGHS-1 KD and PGHS-2 Y385F mutant mice have provided new insights into eicosanoid signaling and will serve as important reagents to elucidate additional distinct roles of the PGHS enzymes in normal physiology and in various pathological states. Platelet activation is a hallmark of severe preeclampsia, and therapeutic intervention with low-dose aspirin in the prevention and treatment of this disease remains controversial. One concern has been that a potential benefit might be confounded by an impact of PGHS-1 inhibition on parturition. This research has shown that inhibition of platelet activation in vivo can be attained while retaining reproductive integrity in PGHS-1 KD mice, indicating the feasibility of using low-dose aspirin or selective inhibitors of PGHS-1 to prevent platelet activation in pregnant females without affecting their capacity for a normal delivery process.

The novel genetic model of selective inhibition of PGHS-2 should allow assignment of roles for COX activity of PGHS-2 in the pathogenesis of diseases in a way that does not rely on pharmacological dosing with coxibs. Comparison of PGHS-2 Y385F mutant mice with PGHS-2 null mice suggests that PG formation by PGHS-1/PGHS-2 heterodimers could explain closure of the ductus arteriosus at birth (10). Formation of heterodimers may explain differential tissue sensitivity to NSAIDs and perhaps can offer a novel approach to therapeutic manipulation of prostaglandin formation. Manipulation of essential fatty acid supply to the PGHS-1 KD and PGHS-2 Y385F mouse models should help to reveal new insights into eicosanoid function and signaling.

\section{Acknowledgements}

These studies were supported by grants from the NIH (GM63130), Canadian Institutes of Health Research (MOP-79459) and Heart and Stroke Foundation of Ontario (NA-5828). CD Funk is the holder of a Tier I Canada Research Chair in Molecular, Cellular and Physiological Medicine and a Career Investigator of the Heart and Stroke Foundation of Canada.

\section{References}

1. ISIS-2 (Second International Study of Infarct Survival) Collaborative Group. Randomised trial of intravenous streptokinase, oral aspirin, both, or neither among 17, 187 cases of suspected acute myocardial infarction ISIS2. Lancet 1988; ii: 349-60.

2. Diener HC, Cunha L, Forbes C, Sivenius J, Smets P, Lowenthal A. European Stroke Prevention Study. 2. Dipyridamole and acetylsalicylic acid in the secondary prevention of stroke. J Neurol Sci 1996; 143: 1-13.

3. CAST (Chinese Acute Stroke Trial). Collaborative Group, CAST randomised placebo-controlled trial of early aspirin use in 20000 patients with acute ischaemic stroke. Lancet 1997; 349: 1641-9.

4. Sanmuganathan PS, Ghahramani P, Jackson PR, Wallis EJ, Ramsay LE. Aspirin for primary prevention of coronary heart disease: safety and absolute benefit related to coronary risk derived from meta-analysis of randomised trials. Heart 2001; 85: 265-71. 
5. Patrono C, Coller B, Dalen JE, FitzGerald GA, Fuster V, Gent M, et al. Platelet-active drugs: the relationships among dose, effectiveness, and side effects. Chest 2001; 119: 39-63S.

6. US Preventive Services Task Force. Aspirin for the primary prevention of cardiovascular events: recommendation and rationale. Ann Intern Med 2002;136:157-60.

7. Smith WL, DeWitt DL, Garavito RM. Cyclooxygenases: structural, cellular, and molecular biology. Annu Rev Biochem 2000; 69: 145-82.

8. Yu Y, Cheng Y, Fan J, Chen XS, Klein-Szanto A, FitzGerald GA, et al. Differential impact of prostaglandin $\mathrm{H}$ synthase 1 knockdown on platelets and parturition. J Clin Invest 2005; 115: 986-95.

9. Cheng $\mathrm{Y}$, Wang $\mathrm{M}$, Yu $\mathrm{Y}$, Lawson J, Funk CD, FitzGerald GA. Cyclooxygenases, microsomal prostaglandin E synthase-1, and cardiovascular function. J Clin Invest 2006; 116: 1391-9.

10. Yu Y, Fan J, Chen XS, Wang D, Klein-Szanto AJ, Campbell RL, et al. Genetic model of selective COX2 inhibition reveals novel heterodimer signaling. Nat Med 2006; 12: 699-704.

11. Carmeliet P, Ferreira V, Breier G, Pollefeyt S, Kieckens $\mathrm{L}$, Gertsenstein $\mathrm{M}$, et al. Abnormal blood vessel development and lethality in embryos lacking a single VEGF allele. Nature 1996; 380: 435-9.

12. Meyers EN, Lewandoski M, Martin GR. An Fgf8 mutant allelic series generated by Cre- and Flp-mediated recombination. Nat Genet 1998; 18: 136-41.

13. Nagy A, Moens C, Ivanyi E, Pawling J, Gertsenstein M, Hadjantonakis AK, et al. Dissecting the role of $\mathrm{N}$-myc in development using a single targeting vector to generate a series of alleles. Curr Biol 1998; 8: 661-4.

14. Catella F, FitzGerald GA. Paired analysis of urinary thromboxane $\mathrm{B}_{2}$ metabolites in humans. Thromb Res 1987; 47: 647-56.

15. Reilly IA, FitzGerald GA. Inhibition of thromboxane formation in vivo and ex vivo: implications for therapy with platelet inhibitory drugs. Blood 1987; 69: 180-6.

16. Gross GA, Imamura T, Luedke C, Vogt SK, Olson LM, Nelson DM, et al. Opposing actions of prostaglandins and oxytocin determine the onset of murine labor. Proc Natl Acad Sci U S A 1998; 95: 11875-9.

17. Horton EW, Poyser NL. Uterine luteolytic hormone: a physiological role for prostaglandin $F_{2 \alpha}$. Physiol Rev 1976; 56: 595-651.

18. Sugimoto Y, Yamasaki A, Segi E, Tsuboi K, Aze Y, Nishimura T, et al. Failure of parturition in mice lacking the prostaglandin F receptor. Science 1997; 277: 681-3.
19. Shimokawa T, Kulmacz RJ, DeWitt DL, Smith WL. Tyrosine 385 of prostaglandin endoperoxide synthase is required for cyclooxygenase catalysis. J Biol Chem 1990; 265: 20073-6.

20. Grosser T, Fries S, FitzGerald GA. Biological basis for the cardiovascular consequences of COX-2 inhibition: therapeutic challenges and opportunities. J Clin Invest 2006; 116: 4-15.

21. McAdam BF, Catella-Lawson F, Mardini IA, Kapoor S, Lawson JA, FitzGerald GA. Systemic biosynthesis of prostacyclin by cyclooxygenase (COX)-2: the human pharmacology of a selective inhibitor of COX-2. Proc Natl Acad Sci U S A 1999; 96: 272-7.

22. Armstrong EP, Malone DC. The impact of nonsteroidal anti-inflammatory drugs on blood pressure, with an emphasis on newer agents. Clin Ther 2003; 25: 1-18.

23. Curhan GC, Willett WC, Rosner B, Stampfer MJ. Frequency of analgesic use and risk of hypertension in younger women. Arch Intern Med 2002; 162: 2204-8.

24. Frishman WH. Effects of nonsteroidal anti-inflammatory drug therapy on blood pressure and peripheral edema. Am J Cardiol 2002; 89: 18-25D.

25. Catella-Lawson F, McAdam B, Morrison BW, Kapoor S, Kujubu D, Antes L, Lasseter KC, et al. Effects of specific inhibition of cyclooxygenase-2 on sodium balance, hemodynamics, and vasoactive eicosanoids. J Pharmacol Exp Ther 1999; 289: 735-41.

26. Smith GC. The pharmacology of the ductus arteriosus. Pharmacol Rev 1998; 50: 35-58.

27. Loftin CD, Trivedi DB, Tiano HF, Clark JA, Lee CA, Epstein JA, et al. Failure of ductus arteriosus closure and remodeling in neonatal mice deficient in cyclooxygenase-1 and cyclooxygenase-2. Proc Natl Acad Sci U S A 2001; 98: 1059-64.

28. Nguyen M, Camenisch T, Snouwaert JN, Hicks E, Coffman TM, Anderson PA, et al. The prostaglandin receptor EP4 triggers remodeling of the cardiovascular system at birth. Nature 1997; 390: 78-81.

\section{Ying Yu}

Institute for Translational Medicine and Therapeutics

University of Pennsylvania

42I Curie Blvd, Room 820 BRB II/III

Philadelphia, PA 19104

USA

E-mail: yuying@spirit.gcrc.upenn.edu 